\title{
Una breve historia de la educación contable en Colombia
}

\author{
Jhon Cardona Arteaga ${ }^{1}$
}

\begin{abstract}
Resumen
El presente documento pretende abordar el tema de la educación contable desde el enfoque de habilidades y competencias en el proceso de formación y profesionalización en el marco del conocimiento contable. Se propone como perspectiva en la disciplina contable variables estratégicas y globales de investigación, análisis crítico y participativo con calidades profesionalizantes y competitivas, a partir, de escenarios misionales (y curriculares) de las instituciones de educación formal y no formal.
\end{abstract}

\section{Palabras claves}

Educación contable, habilidades, competitividad, investigación.

1 Contador público de la Universidad de Antioquia, especialista en Finanzas de la Universidad EAFIT. Profesor titular del Departamento de Contaduría y decano de la Facultad de Ciencias Económicas de la Universidad de Antioquia; miembro del Consejo Superior y del Consejo Académico de la misma Universidad; presidente de la Asociación Colombiana de Facultades de Contaduría Pública [ASFACOP], de la Asociación de Contadores de la Universidad de Antioquia, de la Comisión Interamericana de Educación Contable y del Consejo Técnico de la Contaduría Pública; miembro de juntas directivas y asesor de varias entidades comerciales sin ánimo de lucro; director de la Escuela de Gobierno y Políticas Públicas de Antioquia. Actualmente es profesor de la Universidad de Antioquia. Citar como: Cardona, J. (2017). Una breve historia de la educación contable en Colombia. Activos, 15(28), 71-81. DOI: https://doi.org/10.15332/s0124-5805.2017.0028.05 


\title{
A brief bistory of accounting education in Colombia
}

\begin{abstract}
Abstrac
This document aims to address the issue of accounting education from the approach of skills and competencies in the process of training and professionalization in the framework of accounting knowledge. It proposes as perspective in the accounting discipline, strategic and global variables of research, critical and participative analysis with professional and competitive qualities, from missionary (and curricular) scenarios of formal and non-formal education institutions.
\end{abstract}

\section{Keywords}

Accounting education, skills, competitiveness, research.

Muy buenas noches a todos. Especial agradecimiento a las autoridades de la Facultad, a los responsables de la Revista Activos y a los miembros del grupo de investigación Contaduría, Información y Control Social.

Revisar el tema de la educación contable en este coloquio, por lo menos para nosotros, cobra actualidad, porque, como lo decía Hugo Macías, aquí trabajamos por décadas. Hace diez años publiqué este texto que se titula Educación contable: antecedente, actualidad y prospectiva (Monsalve y Cardona, 2007) junto con el compañero Miguel Ángel Zapata Monsalve, que en paz descanse, que tuvo la virtud de que su última acción académica, como evaluador de los programas de Contaduría, tuviera lugar acá, en esta universidad, en la acreditación del Programa de Contaduría; al poco tiempo falleció, pero no fue por los problemas que encontró en la Facultad, sino por otras circunstancias y porque, como lo dijo la profesora Rosa, el aprendizaje y la enseñanza son tortuosos y difíciles, y es verdad que así es. 
Entonces, las reflexiones que yo traigo son solo algunos elementos, como llaman algunos, una especie de "repito", sobre ideas que hay acá en este texto y que, en mi concepto, no han cambiado, siguen siendo válidas e implican reflexiones que deben hacerse permanentemente cada vez que nos presentemos ante un curso, cada vez que estudiemos el currículo, cada vez que involucremos a los investigadores en problemas relacionados con la disciplina contable y digamos que son un poco de apoyo. En los minutos que tenemos, voy a tratar de leer la ideas que he escrito para este efecto y para este coloquio.

Sea lo primero destacar el interés que se ha tenido en las dos últimas décadas por estudiar el tema de la educación contable. En algunos espacios se han constituido grupos de investigación con pretensiones virtuosas y que han acogido la obligación que tanto profesores como estudiantes deben tener en el desarrollo de los factores que son considerados como criterios de evaluación de su calidad. Además, los cambios en la regulación — tan discutida- sobre la educación universitaria han contribuido al avance en esta materia. El hecho de que existan las revistas, los investigadores y la participación de los académicos internacionales en las revistas es, digamos, de lo más importante para la existencia de estas publicaciones. La muestra, que es la revista Contaduría, de la Universidad de Antioquia, creada en 1982, ha tenido vigencia por ese relacionamiento que se ha logrado con esos académicos no latinos, sino del mundo anglosajón y de todo el mundo, que tienen su punto de vista crítico, su perspectiva y que han sido acogidos en esa revista como debe ser.

Adicionalmente, las reformas curriculares de los diferentes programas han considerado como referencia los planes de estudio de organizaciones globales y la aplicación opcional, en nuestro caso, de las normas internacionales de educación contable desarrolladas por la IFAC (2017) después de la emisión de las primeras guías de educación contable como criterios orientadores, en particular, la guía nueve - sobre los antecedentes académicos, la evaluación de la capacidad y experiencia profesional- y la guía 11 - que cobra vigencia actual y que es la guía internacional de educación sobre 
tecnologías de información para contadores profesionales-. Y, como se dijo, el impacto de la tecnología sobre la educación ha sido tan grande que puede cambiar el rótulo de nuestros programas y su orientación, obviamente, contando con los planteamientos críticos que aquí se han expuesto y que permanentemente deben establecerse.

En el anterior sentido, deben analizarse los estudios comparados que se han realizado de programas o escuelas de Contaduría, bien por trabajos de referenciación de los docentes investigadores o por la participación que han tenido los profesionales y docentes del país en los distintos escenarios donde se discuten los problemas de la profesión, sobre todo en un ambiente global. El hecho de que los docentes vayan a esos congresos internacionales no bajo la perspectiva del turismo académico, sino bajo la elemental concepción de la comparación de modelos que existen en otros países, brinda una fuente importante para el mejoramiento del quehacer en los programas de Contaduría.

Debo destacar en este punto nuestra propia experiencia de participación en la Comisión de Educación de la Sociedad Interamericana de Contabilidad (Sociedad Interamericana de Contabilidad, 2017), en la que tuvimos la oportunidad de ejercer la presidencia durante varios años. Allí se tuvo un amplio conocimiento y relación con el organismo encargado de regular las normas sobre educación contable en el mundo, el IASB del IFAC, a inicios de este siglo; y no fueron planteamientos meramente de obediencia, sino planteamientos críticos acerca de la verdadera aplicación de dichos elementos en los contextos en los que nosotros nos movemos particularmente en Latinoamérica.

Por lo anterior, con relación a la primera pregunta orientadora, las competencias y habilidades requeridas para la formación del contador, son las expuestas en esas guías y las normas mencionadas, además de las consideradas en otras orientaciones internacionales, como las que se derivan del informe sobre el Proyecto Tuning para Europa, de 2006, y el Proyecto Tuning para América Latina, Reflexiones y perspectivas de la Educación Superior 
en América Latina (Proyecto Tuning, 2007 y 2017), sobre competencias genéricas y específicas en los distintos programas de educación superior para Europa y América Latina, presentadas en los encuentros de profesores del área contable tanto en Colombia como en otros países.

Llama la atención que cuando hablamos de habilidades y competencias desvirtuamos el tema genérico de la concepción que hay detrás de estos conceptos y más bien nos aplicamos a mirar cuáles son las habilidades y las competencias que traen ese tipo de regulaciones al contexto de los proyectos que se han hecho en Estados Unidos por parte de la APA o de las normas internacionales de educación contable, sin detenernos a pensar cómo los teóricos, como se mencionaba acá, por ejemplo, en el caso del profesor Orozco, tienen una concepción fundamental y adecuada para los análisis que se hagan en ese contexto de las competencias.

En ese mismo sentido, la hasta hoy fallida Resolución 1801 de 2010 del Ministerio de Educación Nacional, sobre características específicas de calidad para los programas de Contaduría, en correspondencia con la Ley 1188 de 2008, en relación con las competencias establecía virtuosamente: "El programa buscará que el contador público adquiera las competencias básicas genéricas y específicas necesarias para localizar, extraer y analizar datos provenientes de diversas fuentes, para llegar a conclusiones con base en el análisis de la información financiera y contable por medio de procesos de comparación, análisis, síntesis, deducción, entre otros, para generar confianza pública con base en un comportamiento ético". Y esa confianza pública, que, como lo vamos a ver más adelante, es la que atraviesa las manifestaciones últimas que se han producido en términos de la educación contable, es la que refleja un interés, un interés social en el comportamiento de ese contador público que formamos. Además, el tema de las competencias en la formación del contador debe estar alineado y está alineado con el resultado que se evalúa en las pruebas Saber Pro ECAES.

Lo anterior trae consigo -en las consideraciones del perfil del egresado y en el contenido de las áreas de formación- la definición de si se trata de formar un generalista o un especialista. En nuestra opinión, cada vez el 
enfoque es más profesionalizante y cerrado, con mayor énfasis en criterios técnicos que en la universalización del conocimiento. El excesivo impacto de la regulación, primero nacional y ahora internacional, ha trazado una ruta equivocada que conduce a la formación de un técnico especializado en detrimento de su visión general del mundo social y de los negocios. La regulación contable aparece, en nuestro concepto, como un detonante en menoscabo de la calidad de la formación contable. Se requiere que el fundamento de argumentación contable asociado con la doctrina esté alineado con unas bases firmes en ciencias sociales y en disciplinas afines a la contabilidad. Cómo se lamenta el distanciamiento de la doctrina de los teóricos de los clásicos en materia contable; hoy, por ejemplo, se privilegia el libro acerca de los estándares de auditoría sobre el libro de filosofía de la auditoría de 1959 (Aguirre, 1996).

Refiriéndonos a la segunda pregunta orientadora, la reflexión sobre los planes de estudio debe ser permanente, en asocio con los diferentes grupos de interés y acudiendo, como ya lo dijimos, a fuentes de carácter nacional e internacional. El ajuste al contexto nacional es obligatorio, así como la consideración de las condiciones territoriales en el caso de aquellas universidades que están orientadas por la estrategia de regionalización, como es el caso de la Universidad de Antioquia; en este caso, debe discutirse si el plan de estudios es diferente al de la sede central o si lo que se requiere es un desarrollo que tenga en cuenta dicho contexto. Sobra decir que, en el caso nuestro, de la Universidad de Antioquia, el currículo es el mismo, el plan de estudios es el mismo, y las consideraciones sobre si el Programa de Contabilidad que se ofrece en las sedes que se encuentran en el sureste de Antioquia o en el Urabá difieren no se contemplan siquiera como un debate.

Se requiere comprender el contexto nacional e internacional en lo social, ambiental, empresarial, legal, económico, político e institucional en que se inscribió el ejercicio profesional, como lo indicaba la ya mencionada Resolución del Ministerio de Educación Nacional. Y aquí hago hincapié en el tema de la formación política. A veces entendemos que la formación política deteriora la capacidad del elemento que estamos formando. Para 
nosotros, entender la política es entender el contexto, es entender lo social. Escuchaba un mensaje del papa Francisco hace poco, dirigido a los sacerdotes, y decía: "Cómo el sacerdote no va a participar en política, cómo no va a ser partícipe activo de lo social", y entendemos cada vez que eso se vuelve y se vuelca significativamente cuando el ser contador interactúa con otros grupos sociales. No se trata, pues, de decirle al contador que milite o no en un determinado partido, no es eso; es entender que, por ejemplo, sea partícipe de la construcción, que participe en la evaluación y la crítica de las políticas públicas del país sobre los planes de desarrollo y de tantos aspectos sobre los cuales debe reflexionar.

Los procesos de investigación, la proyección social, la educación continuada, tanto en la educación no formal como en el posgrado, son parte de los avances propios de los programas de Contaduría, en orden a generar los espacios de proyección para los egresados futuros o actuales.

Las universidades, apoyadas en los avances de la investigación por medio de grupos reconocidos por la comunidad académica, deben avanzar en las propuestas de formación en posgrado con énfasis en maestrías y doctorados. Ya se dolían anteriormente algunos de que en el país no hubiera doctorados en contabilidad y de que los contadores o los profesores tenían que ir a otros países a estudiar sus doctorados. Bienvenida, en ese sentido, esa formación, porque retroalimenta y posibilita los programas de Contaduría, sobre todo los de pregrado, obviamente, haciendo claridad en la participación de quienes tienen doctorado o en la participación que deben tener en el desarrollo del pregrado correspondiente.

El desarrollo de los pregrados debe estar condicionado a los resultados que se deriven de la producción académica investigativa de los posgrados, generar impacto sobre la investigación formativa de los estudiantes, participantes activos en la gestión del plan de estudio. En el campo de la formación continuada, el contador público, tanto de las universidades como de los organismos profesionales, tiene un papel protagónico por jugar diferenciando entre actividades estructuradas y no estructuradas, lo mismo que en cuanto a condiciones de evaluación según el modelo que se haya 
implementado de acuerdo con las normas internacionales de educación contable, tal como ocurre en países referenciados en América Latina, como es el caso de México. México ha establecido la Guía para la implementación de las normas de formación profesional en contaduría, adoptada por el Instituto Mexicano de Contadores Públicos y por la Asociación Nacional de Facultades de Contaduría y Administración (ANFECA) en el año 2014 (ANFECA, 2014); es muy importante mirar este documento, en el que el gremio profesional asociado al Instituto Mexicano de Contadores Públicos se pone de acuerdo con el organismo que agrupa a las universidades y escuelas de contaduría y administración para respaldar, en el contexto que corresponde, la aplicación de esas normas expuestas globalmente, haciendo eco de que se tienen que cumplir los requerimientos que se cumplen igualmente en Estados Unidos y en Canadá, porque, obviamente, México tiene un acuerdo comercial con Estados Unidos y Canadá y no puede desligarse de esas obligaciones, aun en el caso de la aprobación del contador público. Por eso allí existe la certificación del contador público, como más adelante lo vamos a plantear acá, en este escrito.

Sobre esta materia, es pertinente avanzar en el tema de la certificación profesional como un elemento faltante en los criterios globales de comparación internacional. Una consideración final: en el campo de la educación contable colombiana, se considera pertinente desarrollar una estrategia que involucre un horizonte de tiempo que al menos sea de una década. Estamos hablando del proyecto de Plan Decenal de Educación Terciaria, estamos hablando estratégicamente en mi Universidad. Por ejemplo, en este momento se está planteando el plan de desarrollo por diez años para la Universidad, y pensaba: "10 años ahora no está tan loco". Es necesario hacerlo, porque la prospectiva es la que nos brinda esa posibilidad de asegurar y avanzar en estos aspectos.

Esto debería hacerse de manera similar para la Contaduría: deberían fijarse, en un horizonte de 10 años, los criterios para una propuesta de formación contable que requiere el país, realizar un diagnóstico que revele con claridad el contexto educativo y establecer las perspectivas con base 
en los avances de la disciplina y la profesión contable, que conduzcan a la formación de un profesional de clase mundial crítico y participativo, tal como lo están haciendo profesiones como la medicina.

Actualmente, hay debate en el país sobre la formación del médico. En la prensa de esta semana que terminó hubo unas manifestaciones de facultades de Medicina, y entre ellas la nuestra, la Universidad de Antioquia, donde veían, digamos con beneplácito, que hubiera por los menos un movimiento para generar ese espacio de reflexión alrededor del médico que necesita el país o del médico que se debe formar en las facultades de Medicina, habida cuenta del cuestionamiento que hay a muchos programas o a muchas facultades de Medicina en el país y que ustedes han conocido suficientemente.

También el Consejo de Normas Internacionales de Educación Contable del IFAC, propietario y regulador de las normas de educación contable, ha lanzado su estrategia para los próximos cinco años (2017-2021), con un énfasis marcado en la recuperación de la confianza pública de la profesión, menoscabada por los asuntos ya conocidos en el año 2001 y en el año 2008. Invitamos, pues, a que tanto el documento del Instituto Mexicano de Contadores Públicos (ANFECA) como este plan estratégico del Consejo de Normas Internacionales de Educación Contable sean conocidos para que retroalimenten esa posibilidad que tenemos nosotros de avanzar en este aspecto.

Finalmente, y ya pensando un poco más en la profesión, el Instituto Americano de Contadores Públicos Certificados (IFAC) ha realizado un diagnóstico y proyectado los cambios en la profesión contable en Estados Unidos para los próximos años en su documento Horizontes CPA 2025 (AICPA, 2015) como continuación de su anterior estudio, denominado Proyectos Visión CPA, que confluyó en el año 2011; ese proyecto fue conocido a finales de los años noventa del siglo anterior. En estos estudios han participado contadores, empresarios, reguladores y líderes de opinión para examinar las tendencias actuales y emergentes que afectarían a los CPA hasta el año 2025. ¿No creen ustedes que ha llegado la hora para avanzar 
en estas estrategias que pueden ubicar a nuestra profesión contable colombiana en el lugar que le corresponde? ¿No creen ustedes que sea factible constituir un comité independiente que piense la profesión y que piense la educación contable para los próximos 10 o 15 años en Colombia y que sea el derrotero sobre el cual se construya ese futuro de ese contador público que necesita el país y que necesita la globalidad?

Termino citando al profesor Adolf Antone: "Nuestra sociedad no es estática, y los cambios en las estructuras sociales han de ser analizados y estudiados, dirigiendo los esfuerzos a actualizar y adaptar nuestra disciplina contable a tales cambios socioeconómicos. Los retos planteados por la sociedad y los reportes contables a ella requeridos pueden determinar la eficacia y el progreso futuro de la disciplina contable. Las respuestas adecuadas pueden gradualmente ser vertidas en la teoría, la estructura conceptual y las prácticas contables". El profesor Adolfo entonces escribió esto para la revista Contaduría Universidad de Antioquia en el año 1986, y creo que las cosas no han cambiado mucho hasta este momento. Muchas gracias.

\section{Referencias}

Aguirre, O. (1996). Enciclopedia de auditoría. Madrid: Cultural de Ediciones.

AICPA. (2015). Horizontes CPA 2025 Recuperado de https://www.aicpa.org/ research/cpahorizons2025/cpahorizonsreport.html

ANFECA. (2014). Guía para la implementación de las normas de formación profesional en contaduría. México, D. F.: ANFECA, Instituto Mexicano de Contadores Públicos.

Congreso de la República de Colombia. (25 de abril de 2008). Ley 1188 de 2008). Por la cual se regula el registro calificado de programas de educación superior y se dictan otras disposiciones. D. O. 46.971. Recuperada de https://www. mineducacion.gov.co/1621/article-159149.html

IFAC. (10 de febrero de 2017). International Accounting Standards Board. Recuperado de https://www.iaesb.org

Monsalve, M. A., y Cardona, J. (2007). Educación contable: antecedente, actualidad y prospectiva. Medellín: Universidad de Antioquia. 
Proyecto Tuning. (2007). Reflexiones y perspectivas de la Educación Superior en América Latina. Informe Final - Proyecto Tuning - América Latina 20042007. Bilbao: Publicaciones de la Universidad de Deusto. Recuperado de http://tuningacademy.org/wp-content/uploads/2014/02/TuningLAIII_ Final-Report_SP.pdf

Proyecto Tuning. (20 de 03 de 2017). Proyecto Tuning América Latina. Recuperado de http://tuning.unideusto.org/tuningal/

Sociedad Interamericana de Contabilidad. (15 de febrero de 2017). Asociación Interamericana de Contabilidad. Recuperado de http://www.contadores-aic. org/index.php?node $=832$ 